NASA Technical Memorandum 86887

(WASA-TA-86887) THE EPEECT OF PLASHA ON

185-11133

SOLAB CELL AKRAI IBC CHARACTEEISTICS (UASA)

$17 \mathrm{P}$ HC $\triangle 02 / \mathrm{A} \quad 101$ CSCL $10 A$

G3/20 Unclas 24385

\title{
The Effect of Plasma on Solar Cell Array Arc Characteristics
}

D. B. Snyder and E. Tyree

Lewis Research Center

Cleveland, Ohio

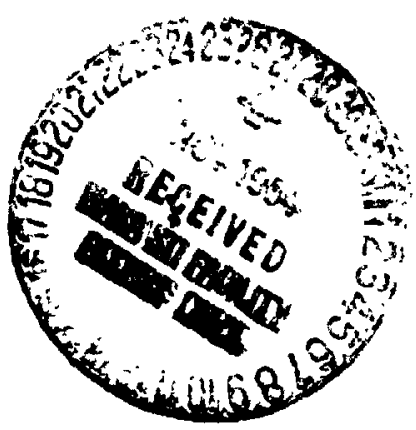

Prepared for the

Twenty-third Aerospace Sciences Meeting sponsored by the American Institute of Aeronautics and Astronautics Reno, Nevada, January 14-17, 1985 
THE EFFECT OF PLASMA ON SOLAR CELL ARRAY ARC CHARACTERISTICS

\author{
D.B. Snyder and E. Tyree* \\ National Aeronautics and Space Administration \\ Lewis Research Center \\ Cleveland, Ohio 44135
}

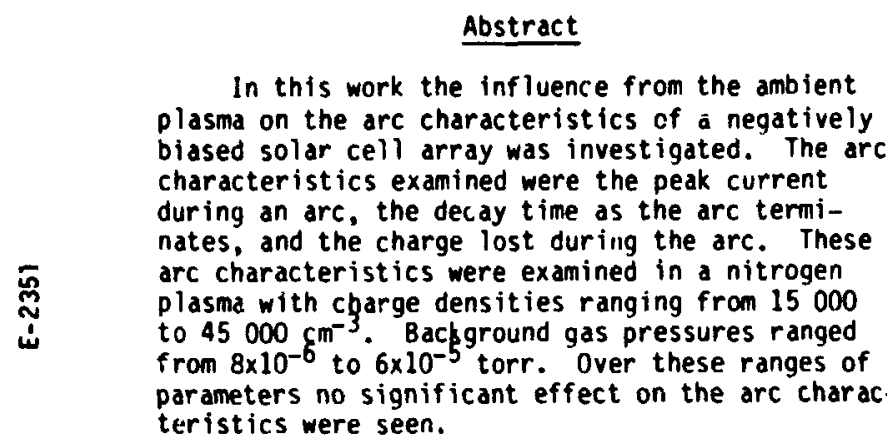

Arc characteristics were also examined for three gas species: helium, nitrogen and argon. The helium arcs have higher peak currents and shorter decay times than nitrogen and argon arcs. There are slight differences in the arc characteristics between nitroqen and argon. These differences may be caused by the differences in mass of the respective species.

Also, evidence is presented for an electron emission mechanism appearing as a precursor to solar array arcs. Occasionally the plasma generator could be turned off, and currents could still be detected in the vacuum system. When these currents are present, arcs may occur.

\section{Introduction}

It is well known that if a shorted dark solar cell array is biased sufficiently negative in the presence of a plasma, it will exhibit arc discharges. 1-4 Characteristic curyes for similar arcs have recently been published, 4-6 and array design considerations have been related to arc characteristics. Three parameters can be used as arc characteristics to describe the current during an arc; the peak current of the arc, the decay time constant of the arc current, and the charge lost during the arc. By obtaining average values and likely ranges for these parameters, the behavior and intensity of the arcs can be compared between various conditions. This paper examines the behavior of solar array arcs in various plasma conditions. This information is useful in predicting the affects arcing will have on high power solar cell arrays in low Earth orbit, and may assist in determining, or at lesst excluding, possible arc mechanisms.

A previous stied found that the arc characteristics do depend on the voltage at which the arc init:ates and on the capacitance between the array and its surroundings. The main purpose of the work reported here is to oetermine whether or not the plasma environment effects the arc characteristics. It is known that the plasma density effects the arc

TresentTy at Case Western Reserve University, Cleveland, on: 0 . rate 8,9 and the threshold above which arcs may occur: 10 however, the effects of plasma on characteristics such as the peak currents of the arcs have not been investigated. The plasma parameters investigated here were the electron density, the neutral density (i.e., pressure), and ion species. While these plasma parameters do not completely specify the plasma, they are the parameters most likely to influence a charge transport mechanism.

The effect of plasma on the arc characteristics is important for two reasons. First, this information is important to the problem of scaling the arcs seen in ground tests to those expected in a low earth orbit environment. If the plasma influences the arc characteristics, then the influence of plasma properties on the range of arc characteristics must be adequately understood to model the effects of solar cell array arcing on high power solar arrays in space. It may be necessary or desirable for spacecraft designers to learn to live with arcs. Data describing the arc characteristics will be useful for designing experiments to further understand the arcs, and to evaluate the risk to spacecraft caused by high voltage systems.

Second, this information provides data useful in understanding the arc mechanism. If the plasma properties influence only the arc frequency and irc threshold voltage and not the arc characteristics, then the plasma is important only in setting up the conditions for arcing to occur and does not participate in the arc mechanism, that is, the actual charge transfer. On the other hand, if a dependence of the arc characteristics on a plasma characteristic is observed, then that plasma characteristic may play a role in the charge transport mechanism of the arc.

Figure 1 shows the time dependence of the current from the array during a typical arc. These arcs are very similar to those reported due to interactions of multi-kiloelectronvolt electron beams with solar cell arrays 5 and similar metalinsulator qeometries. 6 The arc has four time regions. First the arc is initiated and the current increases to a maximum. The rate of increase varies considerably; the rise time varies from less than 0.1 us to on the order of 1 us (Fig. 2). Second, the current chen stays near the peak value for some time. This current may be important in assessing damage to array materials, since resistive heating may cause some material to be boiled off the array. Third, the current decreases with a roughly exponential decay. This decay time is not a measure of the duration of the arc, rather it is only a measure of the duration of the decay portion of the arc. Finally, the arc terminates and the array begins to recharge to the bias voltage. In particular the cover slides may collect substantial amounts of charge from the plasma, resulting in a slight negative pulse. The arc can be described by peak current, the time constant for the decay and the total charge lost during the arc. 
From each arc, three characteristics are obtained: the total charge lost during the arc, the peai current of the arc, and the decay time constant of the arc current as the arc terminates. There is considerable variation in the values of these characteristics among arcs generated under similar conditions (Fig. 2). However, average values of these characteristics for arc populations can be used to show whether or not a suspect paraneter affects their values.

\section{Experiment}

This experiment obtains arc characteristics from a biased solar cell array subjected to a plasma environment. A schematic illustration of the experiment is shown in Fig. 3. A high voltage power supply is used to bias the array at $-1000 \mathrm{~V}$ relative to tank ground. A large resistance decouples the array from the power supply and the connecting cable during an arc by limiting the charge able to flow to the array. The array capacitance to ground was measured to be $350 \mathrm{pF}$. Knowing this capacitance al lows the arc currents to be calculated from the rate of change of array potential. The characteristics of the arcs are determined by using a capacitively coupled probe to measure the rate of change of the solar cell array voltage. A waveform recorder obtains a digitized representation of current to the back plate during an arc for use in later calculations. The net charge lost from the array and the net. current during the arc can be inferred from this information.

The interpretations presented are based on the assumption that the capacitance between the cover slide surfaces and the underlying solar cells is much larger than the capacitance between the cover slide surfaces and the plasma. Under these conditions, when an arc occurs, the potential of the solar cells rises from the negative bias voltage to a potential much closer to plasma ground. The cover slide surfaces begin the arc with a potential near plasma ground. ${ }^{2}$ As the arc progresses the potential of the cover slide surface also rises due to the strong capacitive coupling to the underlying solar rell. The positive cover slide may now collect a return current of electrons from the plasma. At the end of the arc, when the cover slide is most positive this return current may become important (Fig. 2) by the slightly negative signal pulse at the termination of the arc. The currents reported in this paper are the difference between the arc current and the return current to the cover slides; however, the return current is considered to be negligible. Judging from the size of the negative pulse at the end of the arc, the calculations of total charne lost will be within 5 percent of the actual charge lost. The reported arc currents, and the calculated changes in voltage of the array, assume the return current to be zero.

The solar cell array used here has been used in previous arcing studies. 4,5 It is a 24 -cell array segment, constructed of $2 \mathrm{~cm}$ square cells, about $100 \mathrm{~cm}^{2}$ in area. The key feature, for the purposes of this experiment, is a copper plate on the fiberglass support of the array which has a capacitance of $65 \mathrm{pF}$ to the array. This is used as a capacitively coupled probe of the array voltage; the current to this back plate indicates the change in average voltage of the array. The $50 \mathrm{a}$ resistance across the waveform recorder gives a time constant for the cable to the back plate of about
35 ns, much shorter than the time scale of the arcs. This effectively holds the back plate at ground so the transient recorder reads the current to the back plate instead of the voltage of the back plate. This current divided by $65 \mathrm{pF}$ is the rate of change in array voltage.

The $10 \mathrm{Ma}$ carbon resistor decouples the array from the high voltage power supply. This resistance limits the array recharging time to greater than $1.15 \mathrm{~ms}$, much longer than the time scale of arcing. In addition, due to the not-quite-linear I-V characteristic of the resistor, the recharging current is limited to about $0.125 \mathrm{~mA}$, for a voltage drop across the resistor of $1000 \mathrm{~V}$. This current is smaller than the transient recorder can detect at the scale settings used for this work.

There was some concern that the resistor could introduce some extra noise into the arc measurements. To check this, the resistor was placed in series with the 1 input of an oscilloscope. and the $2 \mathrm{~V}$ pulse (Fig. 4) placed across it. No evidence of ringing was detected. Including the high resistance does not introduce noise into the experiment on the same time scale as the arcs.

Including the decoupling resistance introduces some ambiguity into the bias voltage. A steady col]ection current of the array from the plasma of $10^{-5} \mathrm{~A}$ would result in a $100 \mathrm{~V}$ error in the bias voltage. The current collection of the array was not measured in this, work, but it is expected to be less that $10^{-6} \mathrm{~A} .1$

The arc characteristics were measured using two waveform recorders. The data for arcs in helium were taken using a Biomation 610 waveform recorder. This instrument has bandwidth of $2.5 \mathrm{MHz}$ at the scale setting used, smal?er than the decay time constants of the arcs investigated. For ail the other data, a Biomation 6500 waveform recorder was used. This instrument has a much higher bandwidth, but after smoothing the data numerically, the data obtained with this instrument is comparable to that obtained with the Biomation 610 . Data was normally digitized at a rate of $50 \mathrm{~ns} / \mathrm{reading}$. Figure 1 shows a typical arc when the signal is digitized at a rate of $20 \mathrm{~ns} / \mathrm{reading}$. On this time scale considerable ringing is seen with a frequency of about $15 \mathrm{MHz}$. This frequency is characteristic of the cable between the array back plate and the waveform recorder, which rings ot about $12 \mathrm{MHz}$ when the array is pulsed using a square wave generator (Fig. 4). However, since this mode of ringing is excited, the arc may have a frequency component at a higher frequency. The high frequency limit for this experimental arrangement is $12 \mathrm{MHz}$.

To ensure that the relatively slow rise times shown in Fig. 2 are real and not artifacts due to the experimental arrangement, signals due to simulated arcs were examined. Figure 5 shows the response as detected by the back plate. For this test the high voltage power supply was replaced with a power supply set to $-20 \mathrm{~V}$. The arcs were simulated by touching a resistor, with one end grounded, to the array. Two resistors were used: $500 \Omega$ and $1 \mathrm{ka}$. Figure 5(a) shows the resulting signals. In both of these cases, considerable ringing is exhibited as the current immediately rises to its maximum and begins an RC decay. Figure $5(b)$ shows the signais after being filtered numerically using a 0.1 us time constant. The 
decay times measured for these signals are 0.43 us for the $1 \mathrm{ka}$ arc, and $0.28 \mu \mathrm{s}$ for the $500 \mathrm{a}$ arc. These time constants are shorter than those seen in real arcs, and the longer decay times are therefore not artifacts of the experimental arrangement. Comparing the two time constants indicates an internal resistance of $345 \Omega$ and a capacitance of $300 \mathrm{pF}$ rather than the assumed $350 \mathrm{pF}$. The calculated change in voltage, obtained by integrating the current and assuming $350 \mathrm{pF}$, is $13.3 \mathrm{~V}$. In the actual arcs there is less ringing than is seen in the simulated arc. The rise time for the simulated arcs is apparently much faster than for the actual arcs. Thus, the relatively slow rise time seen in the actual arcs is real and not an artifact of the experimental arrangement.

In order to produce more credible peak current measurements, the current histories obtained with the Biomation 6500 were smoothed numerically. A filtering time constant of about 0.1 us produced Satistactory characteristic curves (Fig. 2). Figure 2 shows that there is considerable variation in the characteristics of arcs. The risi time ranges from less than $0.07 \mu \mathrm{s}$ to about 1 us. The peak current and decay times vary over factors of 2 or 3. These variations are real and not simply due to the time scale chosen.

Three arc characteristic parameters were calculated using the digitally recorded history of each arc. The total charge loss was recorded as a change in voltage of the array during an arc. This was calculated by integrating the measured positive current (the recorded negative currents were ignored) over the time of the arc and dividing by the $65 \mathrm{pF}$ capacitance between the back plate and the array. This gives a lower bound on the charge loss since the negative pulse at the arcs termination indicates that there is a small return current to the cover slides of the solar cells during the arc.

The peak current of the arc (ID) was found by simply searching the filtered arc data set for the maximum value of the positive current. The decay time ( $t$ ) of the arc current was calculated using several steps. First, the natural logarithm of the arc current was plotted versus time and a region of the decay where this plot was nearly linear was determined. Next, the slope in that region was determined through a linear least squares fit. The decay time reported is the inverse of that slope.

The plasma source uses electron collisions to ionize gas flowing into the vacuum system. It uses a hot filament to generate electrons. The electrons are accelerated to 50 to $55 \mathrm{~V}$ to ionize gas flowing through the source. A current through a coil concentric around the ionization chamber generates a magnetic field to increase the effective $p^{-1} h$ length of the electrons.

Measurements of the plasma yere obtained throughout the tests. A $1200 \mathrm{~cm}^{2}$ stainless stee l disk (radius $19.5 \mathrm{~cm}$ ) was used as a Langmuir probe. This probe was driven by a saw-tooth generator at a frequency of $1 \mathrm{~Hz}$ from 0 to $15 \mathrm{~V}$. This characteristic curve is digitized and stored every $150 \mathrm{~s}$, to provide a record of the plasma characteristics throughout the $1900 \mathrm{~s}$ test. The electron temperature is typically 1.5 to $2.5 \mathrm{eV}$ and the plasma potential is 4 to $8 \mathrm{~V}$. The charge density ranges from 15000 to $45000 \mathrm{~cm}^{-3}$. There is still some question as to the interpretation of the I-V curves produced from this arrangement. For this reason, the plasma variations are reported in terms of the current collected by the probe at $15 \mathrm{~V}$. This quantity reflects the charge density.

The background pressure may also be adjusted in the pressure range of $8 \times 10^{-6}$ to $6 \times 10^{-5}$ torr. The background gas for these tests is normally nitrogen but tests have been run using both helium and argon as the background gas. The pressures reported are the ion gauge readings during the test and has: not been corrected for variations in collision cross section of the background gas.

\section{Results and Discussion}

\section{Ion Species}

In order to determine whether or not the ion species could affect the arc characteristics, arcs were produced in three gases: helium, nitrogen, and argon. These gases provide an order of magnitude variation in mass. If ion transport is an important mechanism in solar array arcs, systematic effects should have been observed. If chemistry is important to the mechanism then it is expected that arcs might not have been seen in some cases.

The conditions for the reported arc characteristics were reasonably similar for the argon and nitrogen cases. The plasma conditions produced while working with helium are not understood for this set-up. The plasma ionizer did not function properly when helium was used. This will be discussed in more detail below. However, arcs could be generated with helium as a background gas, and their characteristics are included. The background gas pressure for the $\mathrm{N}_{2}$ and ar tests was about $15 \times 17^{-6}$ torr. The plasma density was about $15000 \mathrm{~cm}^{-3}$ for the two gases.

Figure 6 is a plot of peak current, Ip, against decay time, $t$, for the three gases. There is considerable variation in the values of these two parameters; however, the data tend to fall in a region that has a negative slope. This effect may be described by invoking an additional parameter, 7 the product Ipt. This product has units of charge, and reflects the charge lost during the decay portion of the arc. The curves drawn represent the average value of this product for each of the three populations. The Ar and $\mathrm{N}_{2}$ populations overlap, and the difference in Ipt may not be significant. A U-Test 11 indicates a 50 to 60 percent probability that the means for the two populations are the same for Ip and $t$. However, the He population is significantly to the lower left of the $A r$ and $N_{2}$ populations.

The gas species results are summarized further in Table I. The error ranges indicated in the table are standard deviations for the various sets of data. The variations in the Ipt product simply reflect the variations in Ip and $t$, though its distribution is a little more sharply peaked.

The variations in Ip and $t$, while statistically not very significant, show an interesting trend: $t$ increases as mass increases. If $t$ is interpreted as a RC decay time constant, then, since the capacitance is the same for both cases, the resistance, related to the charge transport mechanism, is increasing as the ma'is increases. 
Ip shows the reverse behavior, which is not surprising if the ion mass plays a cole in the charge transport mechanism. It makes sense that less massive ions should be more mobile than more massive ions.

\section{Effects Due to Charge Density}

Figure 7 shows the arc characteristics obtained under various plasma conditions. There is no evidence that the plasma density influences the arc characteristics. In all cases, the slope of a least squares fit line of the data is near zero, and the standard deviation of the slope is much greater than the slope. If plasma conditions affect the arc characteristics, then the effect is weak.

While the plasma does not affect the arc currents significantly, the plasma does affect the arc rate. Figure 8 shows that as the plasma charge density increases, the arc rate also increases. This experiment was not designed to specifically measure arc rates. There normally was a $50 \mathrm{~s}$ dead time introduced as data was dumped from the transient recorder. Those frequencies greater than about $0.01 \mathrm{~Hz}$ :nd less than $0.02 \mathrm{~Hz}$ may be artificially low due :o the dead time. The frequency data reported for rates higher than $0.02 \mathrm{~Hz}$ are real, however, in these cases the transient data was not transmitted and the dead time was less than $0.1 \mathrm{~s}$.

At this time there is still some question as to the plasma conditions during the test. It is believed that the plasma densities ranged from 15000 to $45000 \mathrm{~cm}^{-3}$ (i.e., the charge density varied over a factor of three). However, the shape of the Langmuir Probe I- $y$ curves produced in this arrangement are not sufficiently understood. For the case of the arc rates in plasma the correlation is better between arc rate and the current to the Langmuir probe, than between the arc rate and the calculated plasma.density. For this reason the current to the probe is reported rather than the plasma density.

\section{Effects Due to Pressure}

Figure 9 summarizes the arc characteristics obtained for various background pressures. In this set of figures the average value of each of the three arc characteristics and the Ipt product are plottnd. The error bars represent the standard deviations of data sets containing 4 to 17 values. There are three data sets with only two values. These are the points with the smallest error bars. In these cases the range is plotted rather than the standard deviation. One data set has four values, the rest have 10 or more values apiece. The lines drawn represent the best fit to the average of each data set weighted by the number in each set, while the standard deviation of the slopes are based on the characteristic of all the arcs. These best fit lines are close to horizontal, indicating only weak, if any, dependence on background pressure. Figures 9 (a) and (b) plot charge loss and peak current versus background pressure. No significant dependence is detected. However, the decay time (Fia. $g(c)$ ) shows a dependence which may be significant. This dependence is reflected in the Ipt product (Fig. $g(d)$ ). The decay time tends to decrease as the pressure increases. Despite the large amnunt of scatter in the data, a contribution to the arc current due to collisions of the emitted electrons with the background gas is suggested.

A relationship between pressure and arc rate is suggested by the data (Fig. 10). Arc rates tended to be higher at higher pressures. These data contain the same restrictions as mentioned earlier for the frequency data between 0.02 and $0.01 \mathrm{~Hz}$. Both high $(20.04 \mathrm{~Hz})$ and low $(<0.01 \mathrm{~Hz})$ frequencies were seen at the higher pressures, so ' $t$ is not clear how reliable these data are. The two data points at higher pressures produce the apparent positive slope of the least squares $f$ it line. This apparent dependence of arc rate on pressure may be due to variations in the plasma conditions.

\section{Other Observations}

As was mentioned earlier, the plasma source did not function properly when helium was used as the source gas. This is probably due to the high ionization potential of helium. No plasma could be generated with only the plasma source electronics on. Likewise, if the high voltage supply biasing the solar array was turned on with the plasma source electronics off, no evidence for a charged particle environment could be detected. However, as shown in Fig. 11(a), if both the plasma source electronics and the high voltage power supply to bias the solar array were on, then a charged particle environment was detected. The temperature of the electron distribution appeared to be very high, $10^{\prime} \mathrm{s}$ or $100^{\prime} \mathrm{s}$ of $\mathrm{eV}$, though no attempt to quantify the temperature was made. An electron emission from the array ( $1 \mathrm{keV}$ electrons), producing secondary electrons from the wall, might produce the peculiar electron distribution seen.

Kennerud has observed enhanced current collection on solar arrays associated with arcing:

"The voltage was then reduced to $-10 \mathrm{kV}$ and the plasma current again adjusted to $1 \times 10^{-7}$ amps and allowed to run for 140 hours. During this time it was noticed that even though the plasma current density was never changed, the solar panel current would increase by an order of magnitude (up to $>10^{-6}$ amperes) in a period of about 5 minutes. It would then arc (flashes all over the interconnectors could be seen) and the current would drop back to the originally established value of $1 \times 10^{-7}$ or even lower." 12

This report is similar to the effect seen here with two important differences: it was sometimes, though not always, possible turn off the plasma source and continue to see evidence for the continued existence of a plasma; and currents detected here are electrons collected near the array rather than a positive current collected by the array.

In Fig. 11, the current to a grounded sensor is shown and compared to the times when arcs were detected. The sensor is one of four $10 \mathrm{~cm}^{2}$ aluminum plates located about $2 \mathrm{~cm}$ from each corner of the array, and on the same plane as the array. The sensor is grounded through an electrometer. It is detecting free charge in the vacuum system, rather than the current collected by the solar array. This current may indicate that electrons are being emitted by the array into the vacuum system. 
This emission current was detected for all three gases used in this test with the plasma source turned off. However, only in helium were arcs common. No arcs were observed af ter the plasma source was turned of in argon, and only one arc was detected after several at tempts when nitrogen was used (fig. $11(b)$ ).

The plasma is responsible for setting up the conditions which produce this emission current; but once the emission mechanism is initiated, the plasma only ensures that the emission process can continue. Even with the initiating plasma removed, the emission process can terminate in an arc. The arc probably destroys the conditions that permit emission and, for arcing to continue on the array, a second emission site should exist. The plasma is necessary for arcing to occur, but it does not play a direct role in the arc once it has been initiated.

An electron emission process that is associated with the arcs is consistent with these and another set of observations. It was reported earlier ${ }^{4}$ that the cover slides of the solar cell array are at a more negative potential under conditions when arcing can occur that they are when arcing does not occur. It is expected that the cover slides should normally charge to within a few $k T$ of the plasma potential. This is indeed what hap$p$ ans when arcs cannot occur. But when electron emission from the interconnects is initiated, the cover slides may charge negative. It is only when this happens that arcs can occur.

\section{Conclusions}

In this work the influence of the ambient plasma on arc characteristics of a negatively biased solar cell array was investigated. The arc characteristics examined were the peak current during an arc, the decay time as the arc terminates, and the charge lost by the solar array during the arc. These arc characteristics were determined by analyzing the signal detected by a probe capacitively coupled to the array. This probe detects the rate of voltage change of the array. From this data the time history of the arc current can be inferred.

No significant dependence of the arc characteristics on the plasma parameters of charge density and background pressure were detected. A slight decrease in decay time was observed as the pressure increased. This may indicate that the electrons emitted during the arc can ionize the background gas. This effect is small and the data is noisy. The charge density apparently plays iftle or no role in the determination of the arc characteristics.

Other researchers 8,9 have observed that plasma parameters do affect the arc rate. Increases in charge density result in an increased arc rate. There is some evidence for an increase in arc rate with an increase in pressure, but these data are less conclusive.

Arcs which occur in helium have shorter decay times and slightly higher peak currents than those arcs in argon and nitrogen. It is not clear whether this is due to pressure differences in the experiments or to some other reason. There is weak evfoence that the peak current and decay time of the arcs are related to ion mass. This would be understandable if the lons are the charge carriers during the arc. But the lack of an ion density or pressure dependence does not support this conclusion.

These results are sufficient to advance an initial hypothesis, for the purposes of spacecraf $t$ design, that the ambient plasma will not strongly influence the arc characteristics.

This work should be repeated for a wider range of plasma conditions. It is difficult to obtain conclusive results in this kind of experiment because the arc characteristics vary over a wide range. If these experiments were performed over several orders of magnitude of environmental parameters, then small dependencies on the environment would be evident and the results more conclusive.

It should also be noted that while this work, and related work, result in a fairly consistent set of arc characteristics, all this work has been done on one particular solar cell array. These sorts of measurements should be performed on other solar cell arrays and other solar cell geometries.

In addition to measurements of arc characteristics, this work has produced evidence for an electron emission mechanism, closely related to arcing. The electron emission is a precursor effect to arcing. After emission has begun, an arc may occur. This may provide some clues as to the arc mechanism, and provide suggestions to reduce the impact of solar cell array arcing on spacecraft.

\section{References}

1. Stevens, N.J., "Review of Biased Solar ArrayPlasma Interaction Studies," NASA TM-82693, 1981.

2. Grier, Norman T., and Stevens, N.J., "Plasma Interaction Experiment (PIX) Flight Results," Spacecraft Charging Technology - 1978, NASA CP-2071, 1979, Pp.295-314.

3. Grier, Norman T., "Plasma Interaction Experiment II: Laboratory and Flight Results," proceedings of the Spacecraft Environmental Interactions Conf. 1983, to be published.

4. Snyder, D.B., "Discharges on a Negatively Biased Solar Array in a Charged Particle Environment," NASA TM-83644, proceedings of the Spacecraft Environmental Interactions Conference 1983, to be published.

5. Snyder, D.B., "Environmentally Induced Discharges in a Solar Array," IEEE Transactions on Nuclear Science, Vol. 29, No, 6, Dec. 1982, pp. 1607-1609.

6. Leung, P., "Discharge Characteristics of a Simulated Solar Cell Array," IEEE Transactions on Nuclear Science, Vol. 30, No. 6, Dec. 1983. pp. 4311-4315.

7. S.lyaer, D.B., "Characteristics of Arc Currents on a Negatively Biased Solar Cell Array in a Plasma," NASA TM-83728. 
8. Miller, W.L. "An Investigation of Arc OisCharging on Negatively Biased DielectricConductor Samples in a Plasma," proceedings of the Spacecraft Environmental Interactions Conference 1983, to be published.

9. Ferguson, D., NASA Lewis Research Center, private communication.
10. Stevens, N.J., "Interactions Between Large Soace Power Systems and Low Earth Orbit

Plasmas, "proceedings of the Spacecraft Environmental Interactions Conference 1983, to be published.

11. Bowker, A.H. and Lieberman, G.J., Engineering Statistics, Prentice Hall, Inc., Englewood CTiffs, NJ, 1959, pp. 156-159.

12. Kennerud, K.L., "High Voltage Solar Array Experiments," Boeing Aerospace Company, Seattle WA, Mar. 1974, P. 70. INASA (R-121280). 


\section{ORIGINAL PAGE IS \\ OF POOR QUALITY}

TABLE 1. - THE EFFECT OF GAS SPECIES ON ARC CHARACTERISTICS

\begin{tabular}{|c|c|c|c|}
\hline & Helium & $\begin{array}{l}\text { Nitrogen, } \\
N_{2}\end{array}$ & Argon \\
\hline Mass (amu) & 4 & 28 & 40 \\
\hline lonization potential, a $v$ & 24.5 & 15.6 & 15.4 \\
\hline Pressure ion gauge, $\nu$ torr & $30-60$ & $10-15$ & 15 \\
\hline Peak current, Ip, A & $0.154 \pm 0.043$ & $0.145 \pm 0.023$ & $0.137 \pm 0.045$ \\
\hline Decay time, $t$, us & $0.79 \pm 0.40$ & $1.07 \pm 0.32$ & $1.14 \pm 0.40$ \\
\hline Product, Ipt, ( $\mu$ Coul) & $0.110 \pm 0.033$ & $0.150 \pm 0.029$ & $0.144 \pm 0.033$ \\
\hline $\begin{array}{l}\text { Fraction of charge lost at } \\
-1 \mathrm{kV} \text { bias }\end{array}$ & $0.645 \pm 0.12$ & $0.66 \pm 0.04$ & $0.56 \pm 0.16$ \\
\hline
\end{tabular}

afrom Handbook of Physics and Chemistry, Chemical Rubber Company, 51st Ed. 


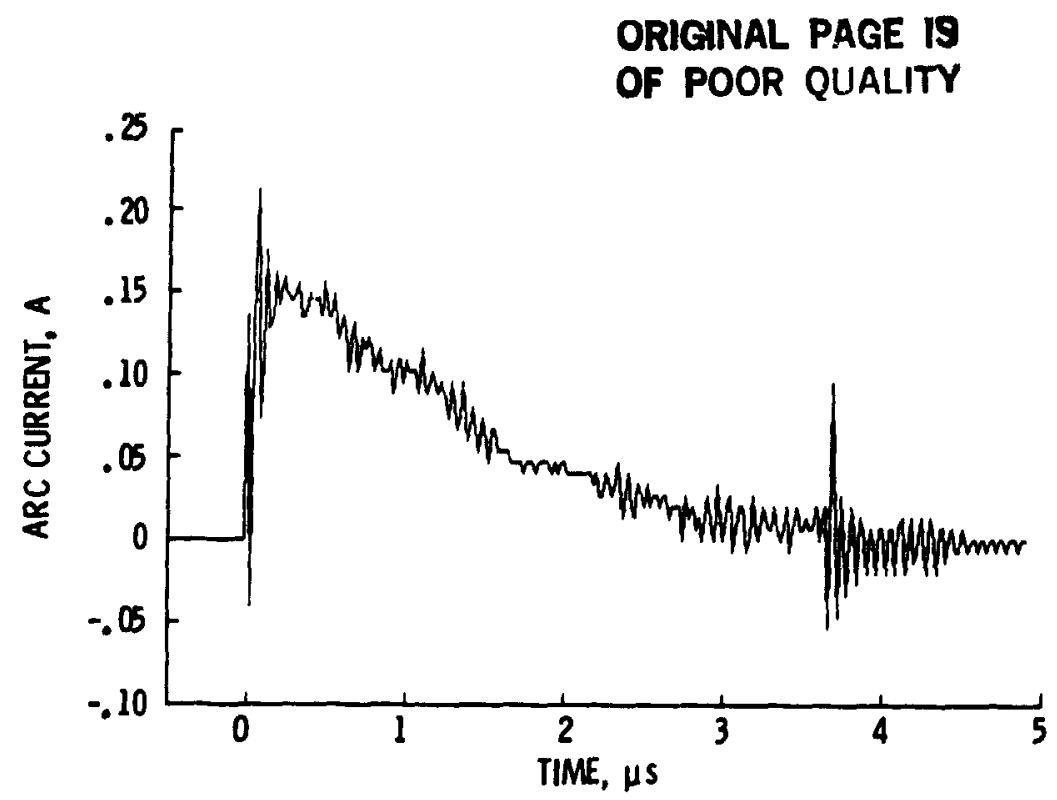

Figure 1. - Typical response to an arc - back plate signal during discharge. Ringing at $15 \mathrm{MHz}$; gas, argon; bias, $-1 \mathrm{kV}$; capacitance, $350 \mathrm{pF}$; digitizing rate, $0.020 \mu \mathrm{s}$ channel.

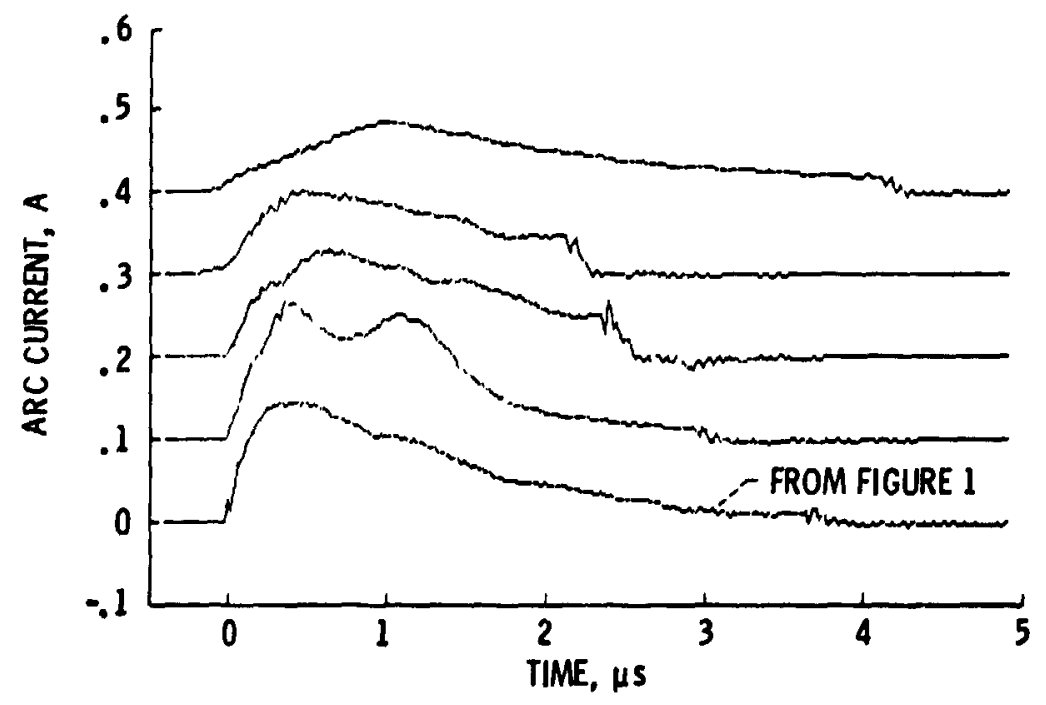

Figure 2. - Varlous solar cell arcs. Signal smoothed by filtering with a time constant of $0.09 \mu \mathrm{s}$. Gas species, argon; blas, $-1 \mathrm{kV}$; capacitance, $350 \mathrm{pF}$. 


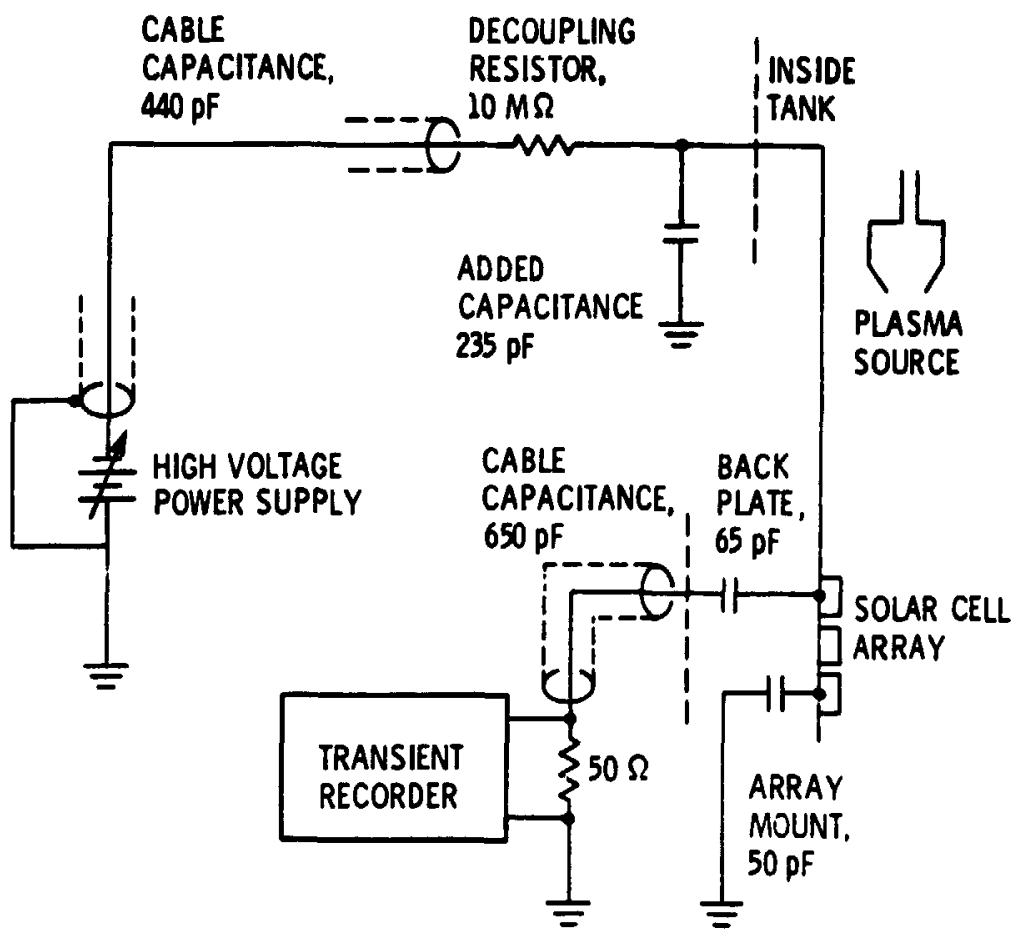

Figure 3. - Schematic diagram of experiment.

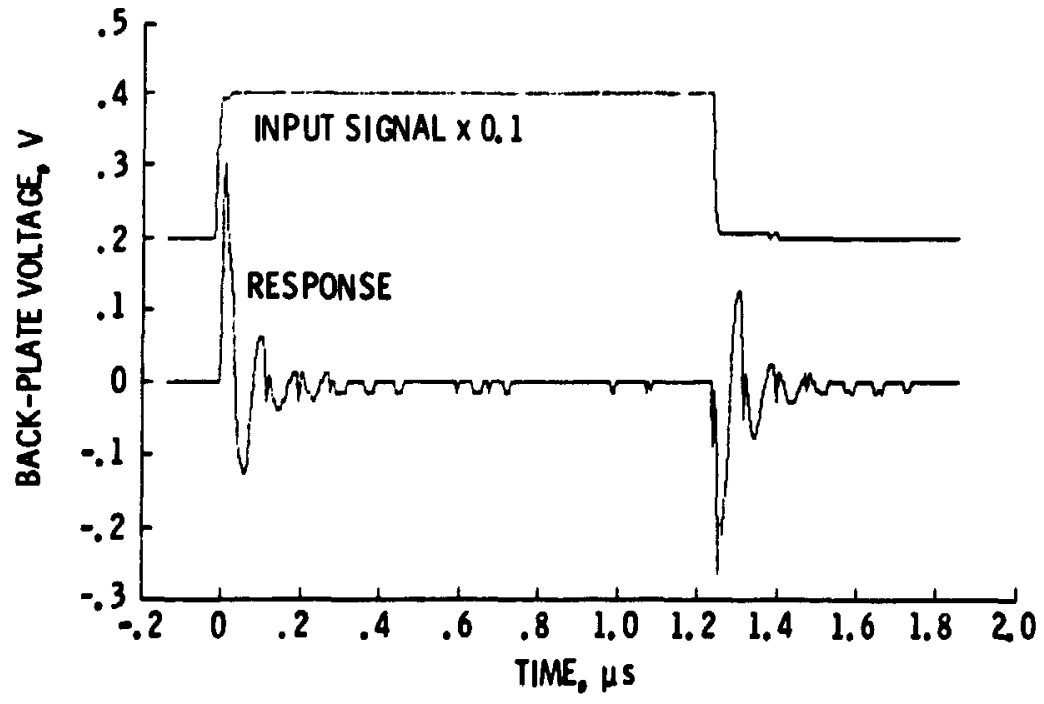

Figure 4 - Back-plate response to a 2-V pulse. Ringing at $\sim 13 \mathrm{MHz}$; capacitance, $115 \mathrm{pF}$; digitizing rate, $0,005 \mu \mathrm{s} /$ channel. 


\section{ORIGINAL PAGE IS}

OF POOR QUALITY

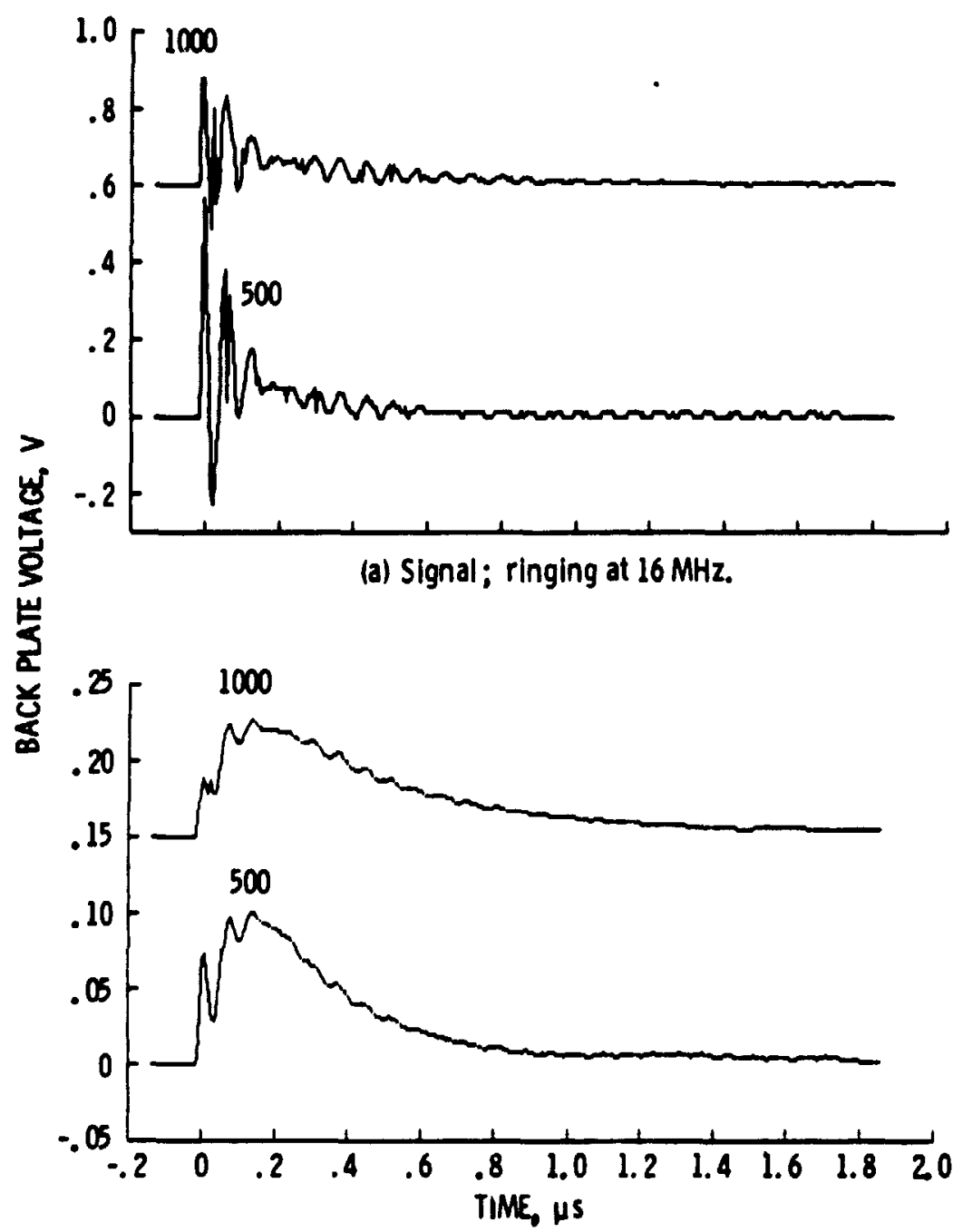

(b) Smoothed signals, filtered with a time constant of $0.01 \mu \mathrm{s}$. 1000-ohm impedence: $13.3-V$ change; $0.43-\mu s$ decay time. 500-ohm impedence: $13.4-V$ change; $0.28-\mu$ s decay time.

Figure 5. - Back-plate response to simulatod arcs. Arc impedances, 2000 and $500 \mathrm{chm}$; blas, $-20 \mathrm{~V}$; capacitance, $350 \mathrm{pF}$; digitizing rate, 5 ns/channel. 


\section{ORIGINAL PAQE IT \\ OF. POOR QUALITY}

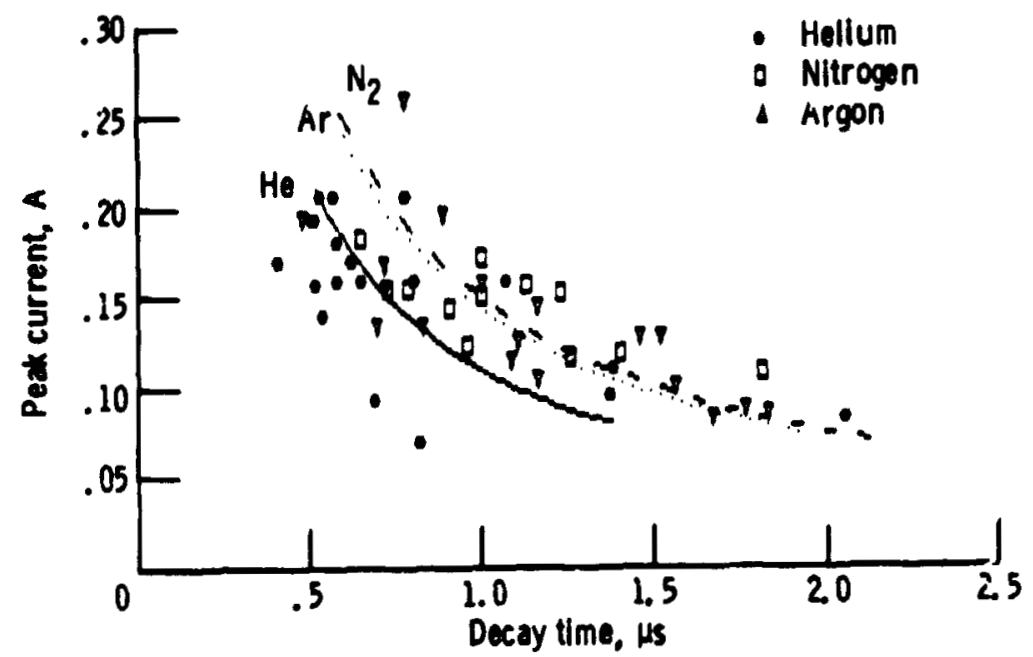

Figure 6, - Dependence of arc chrdracteristics on gas species. Curves represent constant peak current with decay time. $I_{0} \times t$ Average $I_{0} \times t: H e, Q .11 C \mu C: N_{2} Q .250 \mu C ; A r$, Q144; blas, $-1 \mathrm{kV}$; capacitance, $300 \mathrm{DF}$. 


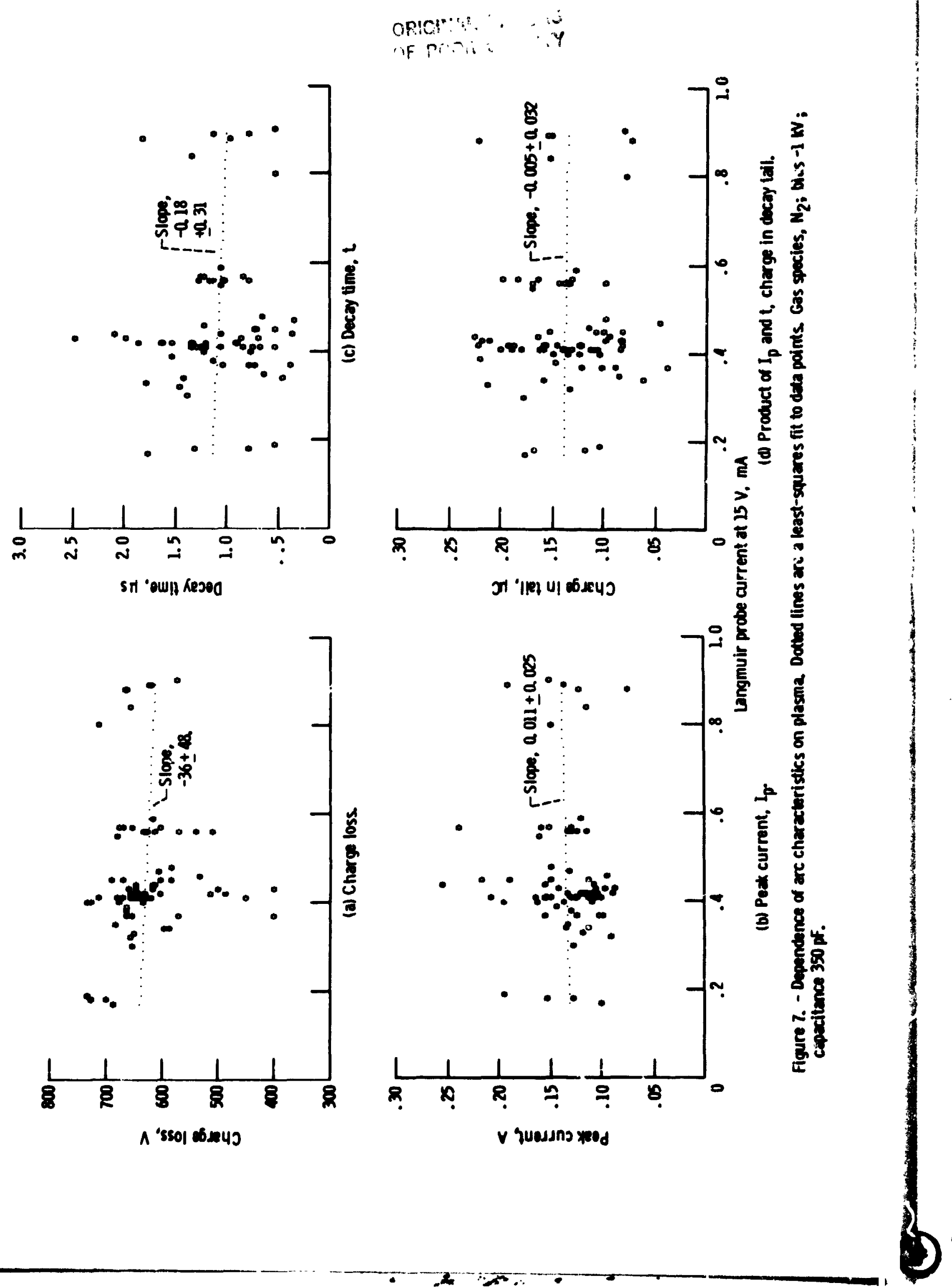




\section{ORIGINAL PACE \\ OF POOR QUALITY}

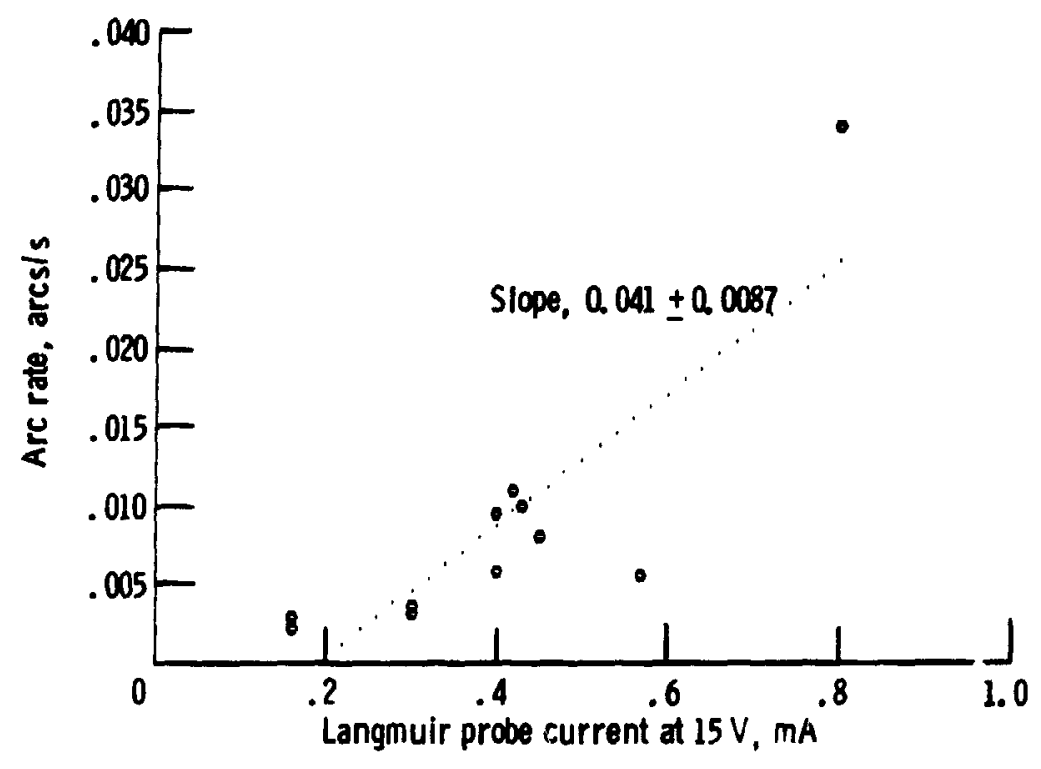

Figure 8. - Dependence of arc rate on plasma. 


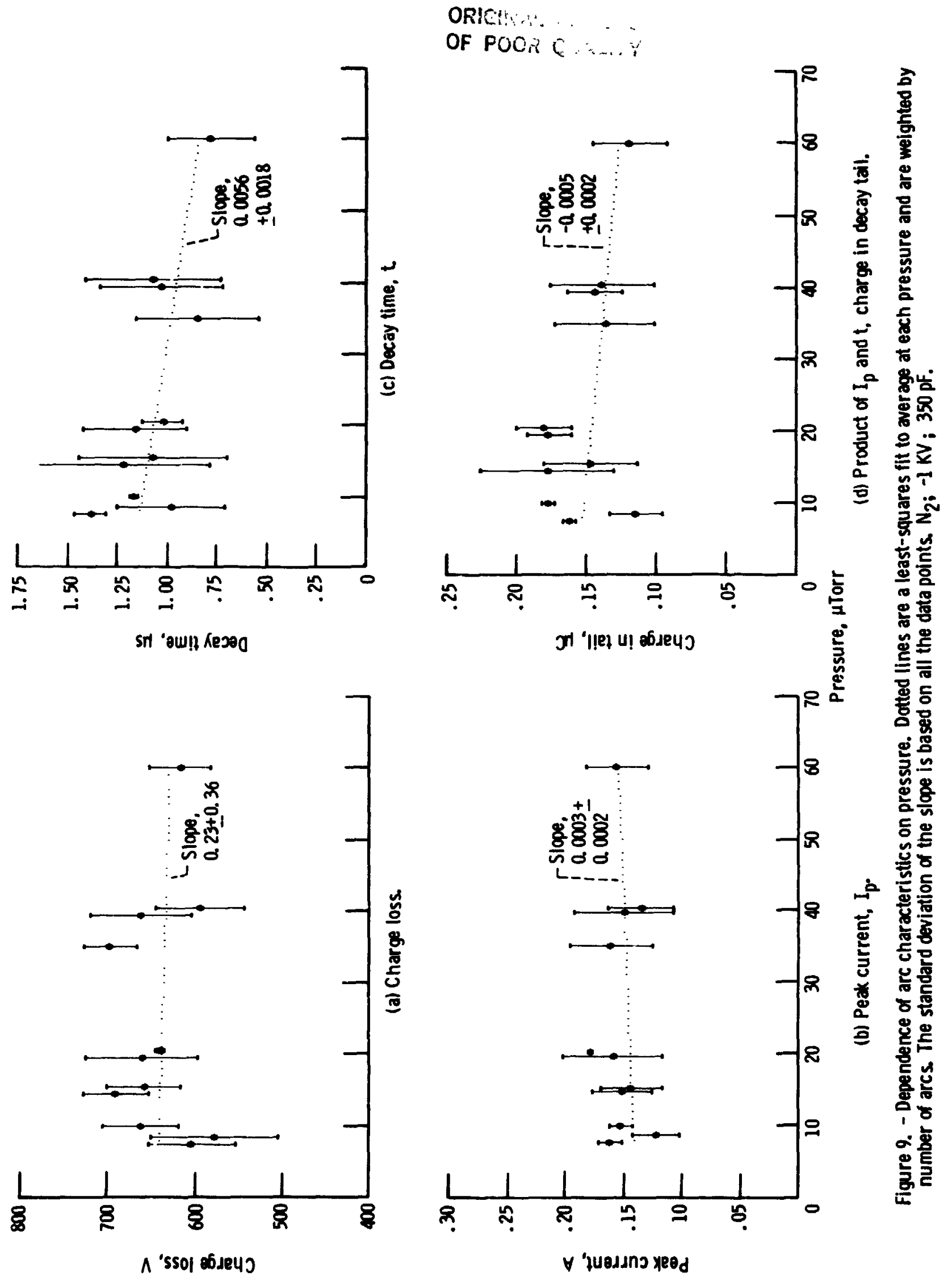




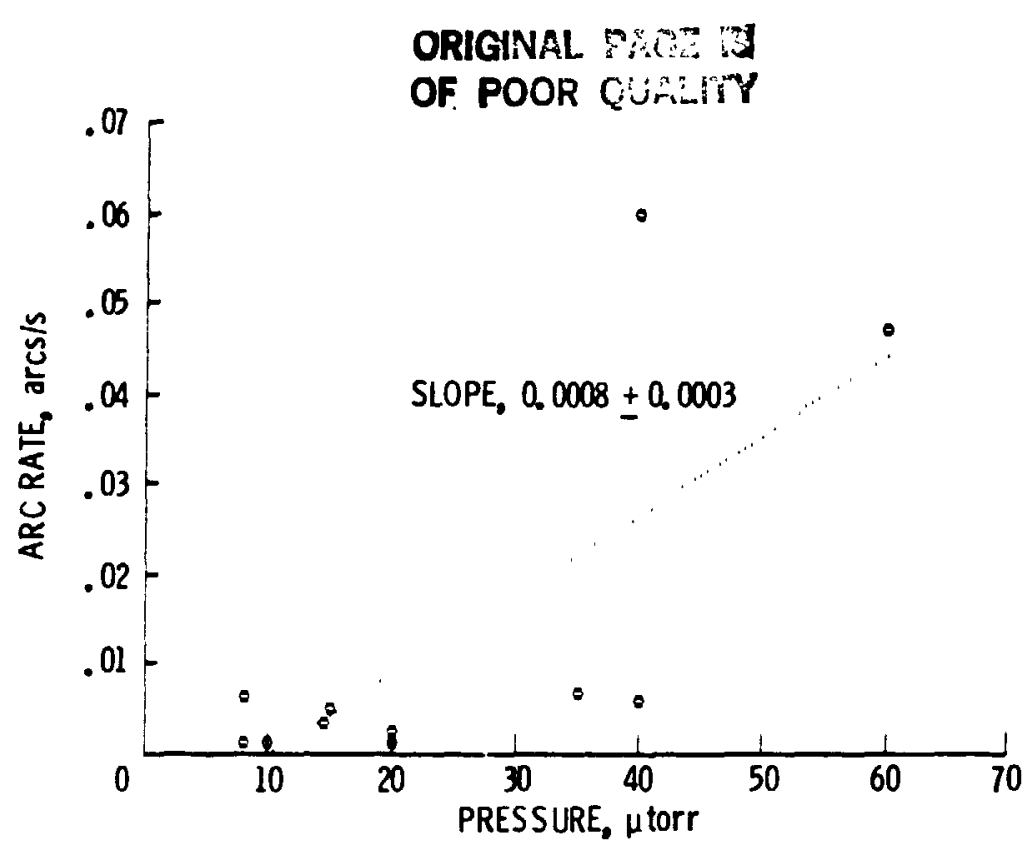

Figure 10. - Dependence of arc rate on pressure. Gas species, $\mathrm{N}_{2}$; bias, $-\mathrm{l} \mathrm{kV}$; capacitance, $350 \mathrm{pF}$.

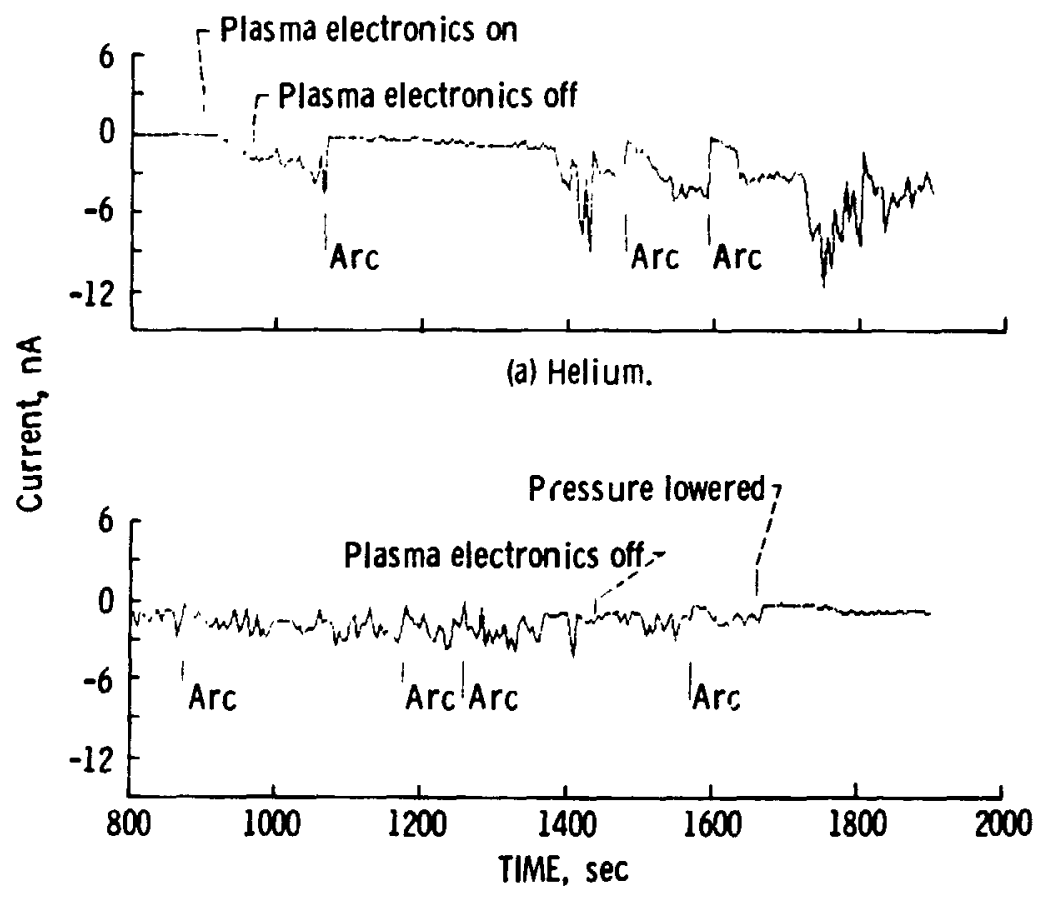

(b) Nitrogen.

Figure 11. - Currents to grounded probe after plas ma source was turned off. Bias, $-1 \mathrm{kV}$; capacitance, $350 \mathrm{pf}$; readings at 5-s intervals. 